\title{
Mechanized Tree Planting in Sweden and Finland: Current State and Key Factors for Future Growth
}

\author{
Back Tomas Ersson ${ }^{1}$ (D), Tiina Laine ${ }^{2}$ and Timo Saksa ${ }^{2, *}$ \\ 1 School of Forest Management, SLU/Swedish University of Agricultural Sciences, \\ 73921 Skinnskatteberg, Sweden; back.tomas.ersson@slu.se \\ 2 Luke/Natural Resources Institute Finland, Latokartanonkaari 9, 00790 Helsinki, Finland; \\ tiinalaine86@gmail.com \\ * Correspondence: timo.saksa@luke.fi; Tel.: +358-29-532-4834
}

Received: 29 March 2018; Accepted: 26 May 2018; Published: 21 June 2018

\begin{abstract}
In Fennoscandia, mechanized tree planting is time-efficient and produces high-quality regeneration. However, because of low cost-efficiency, the mechanization of Fennoscandian tree planting has been struggling. To determine key factors for its future growth, we compared the operational, planning, logistical, and organizational characteristics of mechanized planting in Sweden and Finland. Through interviews with planting machine contractors and client company foresters, we establish that mechanized tree planting in Sweden and Finland presently shares more similarities than differences. Some notable differences include typically longer planting seasons in Sweden, and a tendency towards two-shift operation and less frequent worksite pre-inspection by contractors in Finland. Because of similar challenges, mechanized planting in both countries can improve cost-efficiency through education of involved foresters, flexible information systems, efficient seedling logistics, and continued technical development of planting machines. By striving to have multiple client companies, contractors can reduce their operating radii and increase their machine utilization rates. Above all, our results provide international readers with unprecedented detailed and comprehensive figures and characteristics of Swedish and Finnish mechanized tree-planting activities. We conclude that cooperation between Sweden's and Finland's forest industries and research institutes could enhance the mechanization level of Fennoscandian tree planting.
\end{abstract}

Keywords: tree planting machine; contractor; mechanization; reforestation; silviculture; forestry; Fennoscandia

\section{Introduction}

Despite much effort over the last 50 years, tree planting in Fennoscandia has not successfully been mechanized on a large scale [1-3]. Mechanized tree planting has been shown to be time efficient [4], so the machines can potentially alleviate future labor shortages. In addition, because planting trees mechanically with today's machines produces high-quality regeneration (often with better quality than when trees are planted manually $[2,5,6])$, foresters in both Sweden and Finland are keen on this option, rather than the standard option of manual planting $[7,8]$.

The prevalence of mechanized tree planting is quite low in both countries. In Sweden, the proportion of mechanically planted seedlings historically peaked at circa $12 \%$ during the late 1990s [9] with the highly productive, continuously advancing Silva Nova [10], but fell to $<1 \%$ during the mid-2000s and has stayed there since [2]. In Finland, this figure is considered to be $<5 \%$ [8], despite plenty of research and development work to promote mechanized planting. The proportion of mechanized planting in Finland has, in fact, stagnated or even slightly decreased over the last few years [11]. 
Previous research has highlighted some general reasons why the growth of mechanized tree planting in Fennoscandia is struggling. The main reason for this struggle has primarily been poor contractor profitability leading to reduced interest among contractors [2,3]. Poor profitability is a consequence of mechanized tree planting's relatively low cost-competitiveness, compared to manual tree planting. Cost-competitiveness is low, despite there being a relatively strong demand for mechanically planted trees in both countries [2,4]. Even though the time consumption of mechanized planting is lower compared to separate (spot) mounding followed by manual planting, the cost-efficiency has still been poor compared to manual methods [4]. Cost-efficiency is hampered by low productivity, which originates from operator inexperience, among other reasons [12]. The choice of worksites also strongly influences productivity, and it has been reported that the presence of stones, slash, and stumps decreases the planting machines' productivity [13-17]. The size and spatial distribution of worksites also affects cost-competitiveness [18]. The proportion of time spent relocating the machine decreases as worksite size increases, and the cost and time consumption of relocating increases as the distance between worksites increases $[4,10]$.

Machine Utilization (MU) has a strong impact on contractor profitability, and MU is often comparatively low for planting machines [12]. In turn, factors like workplace organization and seedlings logistics have a strong impact on the planting machines' MU $[10,19,20]$. The theoretical MU of Finnish planting machines has been estimated to be potentially as high as 90\% [21]. However, high MU rates can also potentially cause disadvantages, such as poorer worksite conditions and greater relocation distances.

The client for mechanized planting in Finland is usually a forest company, a local forest owners association (FOA), or a non-industrial private forest owner [8]. A client can be regarded as a service provider, and foresters working for a forest company/FOA are responsible for organizing mechanized planting activities (for example selecting the worksite), while the planting machine contractor is responsible for performing the actual planting work. Non-industrial private forest owners usually buy a planting service from a forest company/FOA rather than directly contract the planting machine contractor themselves. In Sweden, the clients are almost always forest companies; although sometimes, especially in connection to shutdown periods, such as July or winter, planting machines contractors can work directly for non-industrial private forest owners without using a client company as a middleman.

It is the nurseries' responsibility to grow seedlings for mechanized planting. The seedling types used during mechanized planting are generally the same as those used during manual planting [6,22]. In Finland, spring-planted seedlings are usually freezer-stored and packed in cardboard boxes. The summer-planted seedlings (since they are growing and need tending such as watering) are usually packed in open plastic trays, while the autumn-planted seedling are either packed in cardboard boxes or open plastic trays. In Sweden, from spring to autumn, each nursery sticks to only one seedling packaging system [23], and nurseries in southern Sweden stop delivering seedlings in July until the seedlings have hardened enough to withstand packing.

In Finland, mechanized tree planting is more common [3] and thus (despite using the same type of equipment) could be suspected to be relatively more cost-efficient than in Sweden. Accordingly, there might be factors like workplace organization, choice of worksites, and/or seedlings logistics that are coordinated differently within the two countries. Thus, there is a need to analyze how mechanized planting is organized in Sweden and Finland, and to compare and contrast their operational, planning, logistical, and organizational characteristics. This analysis just might identify solutions in one country that can help to increase the cost-competitiveness of mechanized tree planting in another.

The objectives of this study were to: (1) compare mechanized tree planting in Sweden and Finland; (2) identify factors that can increase the cost-competitiveness of Swedish and Finnish mechanized planting; (3) and determine key factors for the future growth of this reforestation tool in these two countries. Because of the business arrangements used in Swedish and Finnish mechanized tree planting, the data collection approach we used was to interview both contractors and client company foresters. 


\section{Materials and Methods}

The development of mechanized planting has been a hot topic both in Finland and in Sweden over the last ten years [24]. Several studies have tried to establish the technical feasibility and effectivity of present planting devices and a couple of dissertations has also been published lately $[2,3]$. As a continuation of these earlier research activities, we conceived the idea to compare the current operational models for mechanized planting in Sweden and Finland.

A semi-structured thematic interview form based on previous studies was prepared for a field visit to southern Sweden conducted in August 2017. During this study trip, the authors had discussions/interviews with a planting machine contractor, with the forest company Södra's foresters at both the district, regional and company level, and with Södra's nursery professionals. These discussions provided the basic information of the current status of (as well as foresters' attitudes towards) mechanized planting in Sweden.

After the field study, four additional Swedish contractors were identified and interviewed (three more in southern Sweden and one in northern Sweden) in autumn 2017. The contractors were identified through contacts with Sweden's major forest companies. In conjunction with these semi-structured contractor interviews, additional semi-structured interviews were also held with those foresters (who were responsible for each planting machine contract) at the district level.

In Finland, some of the corresponding information could be found from an interview study conducted in 2014 [8]. This information was updated via a Metsäteho-maintained online catalogue of Finnish mechanized tree-planting contractors [25], and two typical contractors were interviewed using a semi-structured method during the winter of 2017-2018.

The interview questions were grouped into four categories. The first category concentrated on the operational characteristics and covered questions on equipment, operator, and production. The second category dealt with worksite characteristics like average size, selection criteria, selector, pre-planting inspection, and relocation. The third category comprised characteristics of seedling logistics, including number of planted seedlings, seedling packaging, seedling delivery method, equipment, seedling storage, and tending. The fourth category concentrated on organizational characteristics such as information systems and business arrangements.

The results are presented by showing the typical attributes (i.e., mode/mean for quantitative data, or type for qualitative data) and their range (i.e., variation among the attributes) in both Sweden and Finland. The range of the qualitative data provides an idea of the variation that exists among the contractors. This range was necessary to identify differences in work methods and novel or innovative solutions that may increase planting machine contractor profitability.

\section{Results}

\subsection{Country Comparison}

In general, mechanized tree planting was operationally quite similar in Sweden and Finland (Table 1), although there were some particular exceptions. Most notably, only one brand of planting device was used in Sweden (Bracke Planter), while three brands were used in Finland (Bracke Planter, M-Planter and Risutec); the typical operator experience level was twice as high in Finland as in Sweden (6 seasons vs. 3 seasons); the typical planting season was longer in Sweden than in Finland (7 months vs. 5 months); and two shifts were the norm in Finland, whereas one shift was typical in Sweden. The use of the M-Planter device in Finland led to a higher maximum productivity over a shift ( $360 \mathrm{pl} / \mathrm{PMh}$; $\mathrm{pl}=$ seedlings; $\mathrm{PMh}=$ Productive Machine hour) compared to the maximum reported in Sweden with the Bracke Planter (240 pl/PMh). Typically, operators had no forestry education, although some Swedish operators had secondary forestry education and some Finnish operators had basic forestry education. 
Table 1. Operational characteristics of mechanized tree planting in 2017 in Sweden and Finland ( $\mathrm{pl}=$ seedlings; PMh $=$ Productive Machine hour).

\begin{tabular}{|c|c|c|c|c|c|c|}
\hline \multirow{2}{*}{ Category } & \multirow{2}{*}{ Characteristic } & \multirow{2}{*}{ Attribute } & \multicolumn{2}{|c|}{ Sweden } & \multicolumn{2}{|c|}{ Finland } \\
\hline & & & Typical & Range & Typical & Range \\
\hline \multirow[t]{5}{*}{ Equipment } & Base machine & Type & $\begin{array}{l}\text { Tracked } \\
\text { excavator }\end{array}$ & $\begin{array}{l}\text { Tracked } \\
\text { excavator }\end{array}$ & $\begin{array}{l}\text { Tracked } \\
\text { excavator }\end{array}$ & $\begin{array}{l}\text { Tracked } \\
\text { excavator, } \\
\text { Wheeled } \\
\text { harvester }\end{array}$ \\
\hline & & Mass (t) & 20 & $16-22$ & 16 & $14-22 *$ \\
\hline & Planting device & Type & $\begin{array}{l}\text { Bracke } \\
\text { Planter }\end{array}$ & Bracke Planter & $\begin{array}{l}\text { Bracke } \\
\text { Planter }\end{array}$ & $\begin{array}{l}\text { Bracke Planter, } \\
\text { M-Planter, } \\
\text { Risutec }\end{array}$ \\
\hline & & $\begin{array}{l}\text { Seedling carousel } \\
\text { capacity }(\mathrm{pl})\end{array}$ & 71 & 70-196 & 72 & $60-242$ \\
\hline & & $\begin{array}{l}\text { Number per } \\
\text { contractor }\end{array}$ & 1 & 1 & 1 & $1-2$ \\
\hline \multirow[t]{4}{*}{ Operator } & $\begin{array}{l}\text { Experience level } \\
\text { (years/seasons) }\end{array}$ & $\begin{array}{l}\text { Mechanized } \\
\text { planting }\end{array}$ & 3 & $1-23$ & 6 & $1-24$ \\
\hline & & $\begin{array}{l}\text { Other forestry } \\
\text { experience }\end{array}$ & 20 & $4-40$ & 32 & $3-52$ \\
\hline & & $\begin{array}{l}\text { Other excavator } \\
\text { work }\end{array}$ & 2 & $0-20$ & 3 & $0-20$ \\
\hline & & Forestry education & None & $\begin{array}{l}\text { None, Forestry } \\
\text { secondary } \\
\text { school }\end{array}$ & None & $\begin{array}{l}\text { None, Basic } \\
\text { forestry } \\
\text { education }\end{array}$ \\
\hline \multirow[t]{9}{*}{ Production } & Planting season & Length (months) & 7 & $2-8$ & 5 & $3-6$ \\
\hline & $\begin{array}{l}\text { Productivity } \\
\text { (pl/PMh) }\end{array}$ & $\begin{array}{c}\text { Average over a } \\
\text { year }\end{array}$ & 150 & $120-220$ & 165 & $70-265$ \\
\hline & & $\begin{array}{l}\text { Maximum over } \\
\text { a shift }\end{array}$ & 220 & $180-240$ & 260 & $200-360$ \\
\hline & Target production & Yearly (pl/year) & 150,000 & $30,000-200,000$ & $0 \quad 180,000$ & $70,000-320,000$ \\
\hline & & $\begin{array}{l}\text { Shift-wise } \\
\text { (pl/shift) }\end{array}$ & 1100 & 1000-1900 & 1200 & $1000-1800$ \\
\hline & Average shift & Number per day & 1 & $1-2$ & 2 & $1-2$ \\
\hline & & $\begin{array}{l}\text { Number per } \\
\text { year }\end{array}$ & 150 & $30-200$ & 160 & $55-248$ \\
\hline & & Length (PMh) & 6.5 & $6-8$ & 7 & $6-8$ \\
\hline & $\begin{array}{l}\text { Non-planting } \\
\text { work }\end{array}$ & $\begin{array}{c}\text { Proportion of } \\
\text { base machine's } \\
\text { yearly PMh }\end{array}$ & $20 \%$ & $15-70 \%$ & $38 \%$ & $0-67 \%$ \\
\hline
\end{tabular}

* Data from 2015-2017.

Work sites, planning, and seedling logistics did not vary substantially between the countries either (Tables 2 and 3). However, typical work sites were larger in Finland than Sweden (4.7 ha vs. 3.5 ha); slash-harvested sites were typical in Finland while not in Sweden; contractors in Sweden always pre-inspected the work sites while this habit was rare in Finland; hired truck and trailer was typical when relocating in Sweden while contractor-owned truck and trailer was typical in Finland; relocations were typically faster in Finland than Sweden (1.5 h vs. $2.5 \mathrm{~h}$ ); seedling packaging in Finland also sometimes entailed open plastic trays (during late-summer/early autumn planting); Swedish seedlings were typically stored on planting machines in enclosed metal boxes while Finnish seedlings were typically stored on covered racks. 
Table 2. Work site characteristics of mechanized tree planting in 2017 in Sweden and Finland ( $\mathrm{pl}=$ seedlings). Words enclosed by (brackets) in the 'Range' columns indicate that the circumstance sometimes occurs.

\begin{tabular}{|c|c|c|c|c|c|}
\hline \multirow{2}{*}{ Characteristic } & \multirow{2}{*}{ Attribute } & \multicolumn{2}{|r|}{ Sweden } & \multicolumn{2}{|c|}{ Finland } \\
\hline & & Typical & Range & Typical & Range \\
\hline \multirow[t]{2}{*}{$\begin{array}{l}\text { Average } \\
\text { size }\end{array}$} & Area (ha) & 3.5 & $2-6$ & 4.7 & $2-10$ \\
\hline & $\begin{array}{c}\text { Seedling } \\
\text { prescription (pl) }\end{array}$ & 6500 & 5000-9000 & 8500 & $3600-18,000$ \\
\hline \multirow[t]{2}{*}{$\begin{array}{l}\text { Selection } \\
\text { criteria }\end{array}$} & Requirement & $\begin{array}{l}\text { Mesic to moist } \\
\text { sites; not } \\
\text { too stony/B.Q. } \\
* *<50 \%\end{array}$ & $\begin{array}{l}\text { Mesic to moist sites; } \\
\text { not too stony; slash } \\
\text { harvested; road closer } \\
\text { than } 300 \text { m to edge } \\
\text { of site }\end{array}$ & $\begin{array}{l}\text { Mesic to moist } \\
\text { sites, low } \\
\text { stoniness/B.Q.** } \\
\quad<60 \%\end{array}$ & $\begin{array}{l}\text { Low to medium } \\
\text { stoniness }\end{array}$ \\
\hline & Preference & $\begin{array}{l}\text { Slash } \\
\text { harvested; } \\
\text { site > } 3 \text { ha }\end{array}$ & Site $>1-3$ ha & $\begin{array}{c}\text { Slash } \\
\text { harvested; } \\
\text { site }>4.5 \text { ha }\end{array}$ & $\begin{array}{l}\text { Slash and stumps } \\
\text { harvested; } \\
\text { site }>1-10 \text { ha }\end{array}$ \\
\hline \multirow[t]{2}{*}{ Selector } & of Sites & Forester & $\begin{array}{l}\text { Contractor and/or } \\
\text { forester }\end{array}$ & Forester & $\begin{array}{c}\text { Contractor } \\
\text { and/or forester }\end{array}$ \\
\hline & of Route plan & Contractor & $\begin{array}{l}\text { Forester and/or } \\
\text { contractor }\end{array}$ & Contractor & $\begin{array}{l}\text { Contractor, or in } \\
\text { conjunction with } \\
\text { forester, or forester } \\
\text { only }\end{array}$ \\
\hline $\begin{array}{l}\text { Pre-planting } \\
\text { inspection }\end{array}$ & $\begin{array}{l}\text { Frequency; } \\
\text { Assessor }\end{array}$ & $\begin{array}{l}\text { Always; by } \\
\text { contractor }\end{array}$ & $\begin{array}{l}\text { Always-when in new } \\
\text { area or involving new } \\
\text { foresters; by contractor } \\
\text { or operator }\end{array}$ & $\begin{array}{l}\text { Rarely; by } \\
\text { contractor }\end{array}$ & $\begin{array}{l}\text { Always when new } \\
\text { client company; by } \\
\text { forester or contractor }\end{array}$ \\
\hline \multirow[t]{4}{*}{ Relocation } & Method & $\begin{array}{l}\text { Hired truck \& } \\
\text { trailer }\end{array}$ & $\begin{array}{l}\text { Hired (own) truck or } \\
\text { tractor \& trailer }\end{array}$ & $\begin{array}{l}\text { Own truck \& } \\
\text { trailer }\end{array}$ & $\begin{array}{l}\text { Own(hired) truck or } \\
\text { tractor \& trailer }\end{array}$ \\
\hline & $\begin{array}{l}\text { Average distance } \\
(\mathrm{km})\end{array}$ & 30 & $20-40$ & 22 & $5-60$ \\
\hline & $\begin{array}{l}\text { Maximum distance } \\
\text { from contractor } \\
\text { depot }(\mathrm{km})\end{array}$ & 50 & 20-100 & 62 & $5-125$ \\
\hline & $\begin{array}{c}\text { Average time } \\
\text { consumption (h) }\end{array}$ & 2.5 & $2-3.5$ & 1.5 & $1-3$ \\
\hline
\end{tabular}

** B.Q. = Boulder quota; see [26] for definition.

Table 3. Characteristics of seedling logistics for mechanized tree planting in 2017 in Sweden and Finland ( $\mathrm{pl}$ = seedlings). Words enclosed by (brackets) in the 'Range' columns indicate that the circumstance sometimes occurs.

\begin{tabular}{|c|c|c|c|c|c|}
\hline \multirow{2}{*}{ Characteristic } & \multirow{2}{*}{ Attribute } & \multicolumn{2}{|c|}{ Sweden } & \multicolumn{2}{|c|}{ Finland } \\
\hline & & Typical & Range & Typical & Range \\
\hline \multirow[t]{2}{*}{$\begin{array}{l}\text { Planted } \\
\text { seedling }\end{array}$} & $\begin{array}{c}\text { Type (always } \\
\text { container-grown) }\end{array}$ & Picea abies & $\begin{array}{l}\text { Picea abies seedlings } \\
\text { (and cuttings), Pinus } \\
\text { sylvestris, Larix spp. }\end{array}$ & $\begin{array}{c}\text { Picea abies, } \\
\text { Pinus sylvestris }\end{array}$ & $\begin{array}{c}\text { Picea abies, Pinus } \\
\text { sylvestris, Betula } \\
\text { pendula }\end{array}$ \\
\hline & $\begin{array}{c}\text { Average size } \\
\text { (stem length/root } \\
\text { plug volume) }\end{array}$ & $30 \mathrm{~cm} / 93 \mathrm{~cm}^{3}$ & $\begin{array}{c}20-35 \mathrm{~cm} / 50-120 \\
\mathrm{~cm}^{3}\end{array}$ & $25 \mathrm{~cm} / 85 \mathrm{~cm}^{3}$ & $15-30 \mathrm{~cm} / 50-115 \mathrm{~cm}^{3}$ \\
\hline \multirow[t]{2}{*}{$\begin{array}{c}\text { Seedling } \\
\text { packaging }\end{array}$} & Type & Cardboard box & Cardboard box & $\begin{array}{l}\text { Cardboard box } \\
\text { and/or Open } \\
\text { plastic tray }\end{array}$ & $\begin{array}{l}\text { Cardboard box } \\
\text { and/or Open plastic } \\
\text { tray }\end{array}$ \\
\hline & $\begin{array}{l}\text { Capacity } \\
\text { (pl/unit) }\end{array}$ & 165 & $80-500$ & 130 & $80-250$ \\
\hline $\begin{array}{l}\text { Seedling } \\
\text { delivery }\end{array}$ & Frequency & Weekly & $\begin{array}{l}\text { From weekly to } \\
\text { twice per season }\end{array}$ & Twice a month & $\begin{array}{l}\text { From every few days } \\
\text { to once per month }\end{array}$ \\
\hline
\end{tabular}


Table 3. Cont.

\begin{tabular}{|c|c|c|c|c|c|}
\hline \multirow{2}{*}{ Characteristic } & \multirow{2}{*}{ Attribute } & \multicolumn{2}{|c|}{ Sweden } & \multicolumn{2}{|c|}{ Finland } \\
\hline & & Typical & Range & Typical & Range \\
\hline & Waypoints & $\begin{array}{c}\text { Nursery—contractor's } \\
\text { depot—roadside } \\
\text { depot }\end{array}$ & $\begin{array}{c}\text { Nursery_(contractor's } \\
\text { depot)_roadside } \\
\text { depot }\end{array}$ & $\begin{array}{l}\text { Nursery- } \\
\text { contractor's } \\
\text { depot- } \\
\text { roadside depot }\end{array}$ & $\begin{array}{c}\text { Nursery-client's } \\
\text { depot or contractor's } \\
\text { depot-roadside } \\
\text { depot }\end{array}$ \\
\hline & Deliverer & $\begin{array}{l}\text { Nursery-contracted } \\
\text { courier to } \\
\text { contractor's depot; } \\
\text { contractor to } \\
\text { roadside depot }\end{array}$ & $\begin{array}{l}\text { Contractor or } \\
\text { Nursery-contracted } \\
\text { courier attends to the } \\
\text { whole delivery }\end{array}$ & $\begin{array}{l}\text { Nursery- } \\
\text { contracted } \\
\text { courier to } \\
\text { contractor's } \\
\text { depot }\end{array}$ & $\begin{array}{l}\text { Nursery-contracted } \\
\text { courier to client's } \\
\text { depot or roadside } \\
\text { depot }\end{array}$ \\
\hline \multirow[t]{2}{*}{$\begin{array}{l}\text { Contractor- } \\
\text { owned } \\
\text { equipment }\end{array}$} & $\begin{array}{l}\text { Seedling storage } \\
\text { at contractor's } \\
\text { depot }\end{array}$ & Cooler storage & $\begin{array}{l}\text { Uncooled storage } \\
\text { hall or underground } \\
\text { cellar or purchased } \\
\text { (rented/shared) } \\
\text { cooler storage }\end{array}$ & $\begin{array}{l}\text { Semi-cooled } \\
\text { storage }\end{array}$ & $\begin{array}{l}\text { Uncooled storage } \\
\text { hall or underground } \\
\text { cellar or purchased } \\
\text { (rented/shared) } \\
\text { cooler storage }\end{array}$ \\
\hline & $\begin{array}{l}\text { Secondary } \\
\text { seedling } \\
\text { transport }\end{array}$ & $\begin{array}{l}\text { Covered pickup } \\
\text { truck }\end{array}$ & $\begin{array}{l}\text { Covered pickup } \\
\text { truck or } \\
\text { covered(open) trailer }\end{array}$ & $\begin{array}{c}\text { Covered } \\
\text { pickup truck }\end{array}$ & $\begin{array}{l}\text { Van or trailer or } \\
\text { pickup truck }\end{array}$ \\
\hline \multirow[t]{2}{*}{$\begin{array}{l}\text { Storage on } \\
\text { planting } \\
\text { machine }\end{array}$} & Type & $\begin{array}{l}\text { Enclosed metal box } \\
\text { on the side of the } \\
\text { crane pillar }\end{array}$ & $\begin{array}{l}\text { (Ground-accessible) } \\
\text { enclosed metal box } \\
\text { on the side of the } \\
\text { crane pillar }\end{array}$ & $\begin{array}{l}\text { Covered or } \\
\text { open rack on } \\
\text { the back of the } \\
\text { excavator }\end{array}$ & $\begin{array}{l}\text { Covered or open } \\
\text { rack on the base } \\
\text { machine's back side, } \\
\text { enclosed metal box } \\
\text { on the side of the } \\
\text { crane pillar }\end{array}$ \\
\hline & Capacity (pl) & 1800 & $1100-3000$ & 1800 & $1000-4000$ \\
\hline \multirow[t]{2}{*}{$\begin{array}{l}\text { Seedling } \\
\text { tending }\end{array}$} & Activities & Shading & $\begin{array}{l}\text { Shading; watering; } \\
\text { unstacking or } \\
\text { moving (opening) } \\
\text { boxes }\end{array}$ & Shading & $\begin{array}{l}\text { Shading; watering; } \\
\text { unstacking or } \\
\text { moving (opening) } \\
\text { boxes }\end{array}$ \\
\hline & $\begin{array}{l}\text { Average time } \\
\text { consumption } \\
\text { (min/shift) }\end{array}$ & 30 & $0-30$ & 30 & $0-60$ \\
\hline
\end{tabular}

The typical information systems and business arrangements for mechanized tree planting were also quite similarly organized between Sweden and Finland (Table 4). Indeed, in both countries, work orders were typically to be delivered by the client company via an internet application minimum 2-2.5 months before planting. This application was also typically used to report the quality control sampling (usually with a sampling frequency of circa two plots per ha). Piece-rate remuneration was typical in both countries, although there was sometimes area-based payment in Finland.

Table 4. Organizational characteristics of mechanized tree planting in 2017 in Sweden and Finland (pl = seedlings).

\begin{tabular}{|c|c|c|c|c|c|c|}
\hline \multirow{2}{*}{ Category } & \multirow{2}{*}{ Characteristic } & \multirow{2}{*}{ Attribute } & \multicolumn{2}{|c|}{ Sweden } & \multicolumn{2}{|c|}{ Finland } \\
\hline & & & Typical & Range & Typical & Range \\
\hline \multirow[t]{2}{*}{$\begin{array}{l}\text { Information } \\
\text { Systems }\end{array}$} & $\begin{array}{l}\text { Work order } \\
\text { (instructions } \\
\text { and map) }\end{array}$ & $\begin{array}{l}\text { Delivery } \\
\text { method }\end{array}$ & $\begin{array}{c}\text { Internet } \\
\text { application }\end{array}$ & $\begin{array}{c}\text { Visit to } \\
\text { landowner } \\
\text { or paper or } \\
\text { email or } \\
\text { Internet } \\
\text { application }\end{array}$ & $\begin{array}{c}\text { Internet } \\
\text { application }\end{array}$ & $\begin{array}{l}\text { Paper or email } \\
\text { or Internet } \\
\text { application }\end{array}$ \\
\hline & & $\begin{array}{l}\text { Delivery } \\
\text { deadline }\end{array}$ & $\begin{array}{l}2 \text { months } \\
\text { before } \\
\text { arrival to } \\
\text { site }\end{array}$ & $\begin{array}{l}\text { 0.5-4 } \\
\text { months } \\
\text { before } \\
\text { arrival to } \\
\text { site }\end{array}$ & $\begin{array}{l}2.5 \text { months } \\
\text { before } \\
\text { arrival to site }\end{array}$ & $\begin{array}{l}0.5-5.5 \text { months } \\
\text { before arrival } \\
\text { to site }\end{array}$ \\
\hline
\end{tabular}


Table 4. Cont.

\begin{tabular}{|c|c|c|c|c|c|c|}
\hline \multirow{2}{*}{ Category } & \multirow{2}{*}{ Characteristic } & \multirow{2}{*}{ Attribute } & \multicolumn{2}{|c|}{ Sweden } & \multicolumn{2}{|c|}{ Finland } \\
\hline & & & Typical & Range & Typical & Range \\
\hline & $\begin{array}{l}\text { Seedling } \\
\text { ordering }\end{array}$ & Order method & $\begin{array}{c}\text { Manually } \\
\text { via forester }\end{array}$ & $\begin{array}{l}\text { Manually } \\
\text { via forester, } \\
\text { Pre-season } \\
\text { clump } \\
\text { order }\end{array}$ & $\begin{array}{c}\text { Manually } \\
\text { via forester }\end{array}$ & $\begin{array}{c}\text { Straight from } \\
\text { nursery } \\
\text { via contractor } \\
\text { or forester, } \\
\text { Pre-season } \\
\text { clump order }\end{array}$ \\
\hline & & $\begin{array}{l}\text { Minimum } \\
\text { timespan from } \\
\text { order to } \\
\text { roadside depot }\end{array}$ & $\begin{array}{l}8 \text { work } \\
\text { days }\end{array}$ & $\begin{array}{l}0-10 \text { work } \\
\text { days }\end{array}$ & 4 work days & $0-7$ work days \\
\hline & Quality control & $\begin{array}{l}\text { Reporting } \\
\text { method }\end{array}$ & $\begin{array}{l}\text { Internet } \\
\text { application }\end{array}$ & $\begin{array}{l}\text { None or } \\
\text { Paper } \\
\text { forms or } \\
\text { Email or } \\
\text { Internet } \\
\text { application }\end{array}$ & $\begin{array}{c}\text { Internet } \\
\text { application }\end{array}$ & $\begin{array}{c}\text { None or Paper } \\
\text { forms or Email } \\
\text { or Internet } \\
\text { application }\end{array}$ \\
\hline & & $\begin{array}{l}\text { Onsite sampling } \\
\text { frequency }\end{array}$ & $\begin{array}{c}\text { Two } 25 \mathrm{~m}^{2} \\
\text { sample } \\
\text { plots per } \\
\text { ha }\end{array}$ & $\begin{array}{l}\text { One-three } \\
25-50 \mathrm{~m}^{2} \\
\text { sample } \\
\text { plots } \\
\text { per ha }\end{array}$ & $\begin{array}{l}\text { One } 50 \mathrm{~m}^{2} \\
\text { sample plot per } \\
500 / 1000 \mathrm{pl} \\
\text { planted }\end{array}$ & $\begin{array}{c}\text { One-four } \\
50 \mathrm{~m}^{2} \text { sample } \\
\text { plots per ha, } \\
\text { Once per shift }\end{array}$ \\
\hline & $\begin{array}{l}\text { Productivity } \\
\text { follow-up }\end{array}$ & Recipient & Contractor & $\begin{array}{l}\text { None, } \\
\text { Contractor }\end{array}$ & Contractor & $\begin{array}{c}\text { None, } \\
\text { Contractor or } \\
\text { Forester }\end{array}$ \\
\hline \multirow[t]{4}{*}{$\begin{array}{l}\text { Business } \\
\text { Arrangement }\end{array}$} & $\begin{array}{l}\text { Between forest } \\
\text { company and } \\
\text { contractor }\end{array}$ & Remuneration & Piece-rate & $\begin{array}{l}\text { Hourly } \\
\text { compensation; } \\
\text { Piece-rate }\end{array}$ & ; Piece-rate & $\begin{array}{l}\text { Area-based or } \\
\text { hourly } \\
\text { compensation; } \\
\text { Piece-rate }\end{array}$ \\
\hline & & $\begin{array}{l}\text { Number of } \\
\text { client forest } \\
\text { companies }\end{array}$ & 1 & $1-4$ & 1 & $1-3$ \\
\hline & $\begin{array}{c}\text { Marketing } \\
\text { towards } \\
\text { landowners by } \\
\text { forest } \\
\text { companies }\end{array}$ & Method & $\begin{array}{l}\text { Field } \\
\text { demos }\end{array}$ & \begin{tabular}{l}
\multicolumn{1}{c}{ Field } \\
demos; \\
Information \\
dissemination \\
to landowners
\end{tabular} & Field demos & $\begin{array}{l}\text { Field demos; } \\
\text { Information } \\
\text { dissemination } \\
\text { to landowners }\end{array}$ \\
\hline & & Frequency & Annually & $\begin{array}{l}\text { Seasonally } \\
\text { to None }\end{array}$ & Annually & $\begin{array}{l}\text { Seasonally } \\
\text { to Annually }\end{array}$ \\
\hline
\end{tabular}

\subsection{Factors Leading to Cost-Competitive Mechanized Tree Planting}

Based on the data in Tables 1-4, we identified 22 factors that lead to cost-competitive mechanized planting (Figure 1). Cost-competitiveness offers room for contractors to become profitable, and profitable contractors are necessary for the growth of mechanized tree planting in both countries. The 22 factors can be broadly defined as factors that: concern seedlings and the planting result; lower fixed costs; increase Machine Utilization (MU); decrease the negative consequences of relocating; and increase productivity and/or work quality (via, e.g., better site selection). 


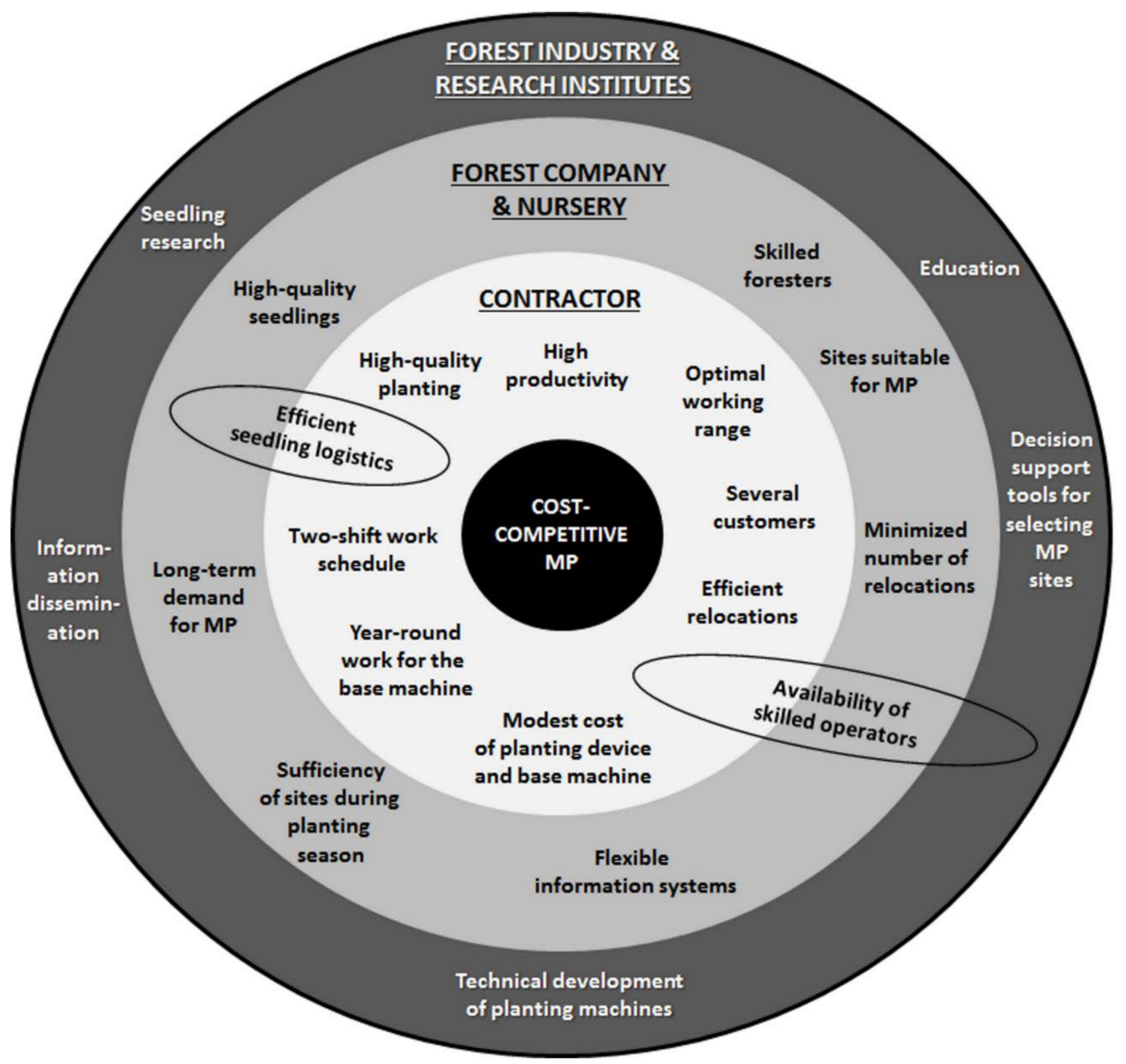

Figure 1. A conceptual framework showing the factors leading to cost-competitive mechanized tree planting (MP) in both Sweden and Finland. The factors in the outer darker ring are those chiefly shaped by the forest industry as a whole and by research institutes. The factors in the middle grey ring are those influenced by the forest company and the nursery supplying the seedlings. The factors in the inner white ring are those mainly affected by the planting machine contractor. The two encircled factors (efficient seedling logistics and availability of skilled operators) are influenced by two or three actors (contractor plus nursery, and contractor, forest company plus whole forest industry, respectively). Modified from reference [27].

\subsection{Key Factors for Future Growth of Mechanized Tree Planting}

Factors that were judged to be key (i.e., the lowest-hanging fruit) for the future growth of mechanized tree planting in both countries were as follows:

1. Education of foresters (to generate competent selectors of work sites, and acceptance of planting machines as a reliable reforestation tool), information to landowners (to create a higher demand for mechanized tree planting leading to a greater selection of suitable sites while reducing the working radius and increasing autumn planting opportunities), and education of operators (to ensure they know the best work methods leading to maximum productivity).

2. Flexible information systems that can help identify suitable planting sites, increase Machine Utilization (MU) through easier administration and seedling ordering, and increase the accuracy of follow-ups (e.g., using Risutec's ASTA system [28] or something similar). 
3. Efficient logistics of suitable seedlings (which increases MU, and ensures high seedling vitality and post-planting performance).

4. High-quality planting work (which ensures continued demand for mechanized tree planting so that landowners receive added value as compensation for the machines' present-day higher planting costs vs. manual planting).

5. Contractors having several client forest companies as this arrangement supports efficient route planning, spreads risk and helps contractors leverage themselves against the (often so) larger forest companies, and reduces the working radius (which leads to, e.g., shorter commutes for the operator).

6. Continued technical development of planting machines (so as to ensure higher quality plantings, higher machine productivity, and/or lower costs in the future).

\section{Discussion}

According to our findings, mechanized tree planting in Sweden and Finland presently share more similarities than differences. Nonetheless, some Finnish contractors had two planting machines, while all Swedish contractors had only one. Also, Finnish contractors more often had two client companies and operated in two shifts per day, while Swedish contractors typically only had one client company and operated in a single shift per day. Still, annual use [29,30] (or more specifically Capacity Utilization [31] or Total Utilization [12]) of the Swedish planting machines was typically almost the same as the Finnish machines (in terms of average number of shifts per year: 150 vs. 160; Table 1) because of southern Sweden's longer planting season (typically 7 months vs. 5 months; Table 1).

In comparison to those of Fennoscandian harvesting contractors, our identified key factors share some similarities, like the need for route efficiency [32] and for contractors to find ways to leverage themselves against the larger client forest companies [33]. Our key factors "High quality planting work" and "Education of operators" have previously been identified by Laine et al. [8] as critical success factors for Finnish mechanized tree-planting contractors. Meanwhile, Mäkinen [34] concluded that for Finnish harvesting contractors, having only one client was a success factor. This is contrary to our key factor "Contractors having several client forest companies", but the harvesting contractors in Mäkinen's study worked year around and were reported to have Capacity Utilization rates up to $99 \%$. Planting machines, in Finland especially, cannot work year around, and tend to have one-third as high Capacity Utilization rates [12]. Thus, during the short Fennoscandian planting seasons, efficient route planning and small operating radii (the latter which multiple clients can give rise to) become comparatively more important for planting machines if they are to secure enough productive hours to pay for their capital costs.

Swedish contractors always pre-inspected work sites, while Finnish contractors rarely did (Table 2). If the contractor is also the sole operator (as was the case for 3 of 5 Swedish contractors), contractor pre-inspection reduces the planting machines' number of productive machine hours (or at least their Machine Utilization rates), which is detrimental to their cost-efficiency. The lesser need for pre-inspection by Finnish contractors is probably a result of the Finnish foresters' comparatively greater experience in selecting sites suitable for mechanized planting (mechanized planting being at least five times more common in Finland than in Sweden).

In Finland, land-owning forest companies like UPM have embraced mechanized tree planting [3]. In Sweden, mechanized planting is demanded by non-industrial private forest owners but not by land-owning forest companies. This difference in acceptance might explain why Swedish contractors typically only had one client company, while Finnish contractors more often had two.

Efficient seedling logistics increases machine uptime, which is in turn a prerequisite for profitable mechanized tree planting [35]. Efficient seedling logistics requires cooperation between the forest company and contractor (Figure 1), but the terms of cooperation might be wholly dictated by the (generally) larger forest company (cf. Reference [36]), leading to contractor frustration and poor motivation. Nevertheless, simply understanding that efficient seedling logistics is also a responsibility of the forest company might lead to greater efforts by the forest company [18]. 
Similar to all mechanized forestry work [37,38], the operator's skill level has a profound effect on planting machine productivity [12,14]. Thus, profitable mechanized tree planting requires access to skilled operators. However, the onus of training operators to become skilled cannot be strictly delegated to the contractors (Figure 1). Instead, this responsibility must also be shared with the whole forest industry, as is done with the training of Fennoscandian harvester and forwarder operators $[39,40]$. Individual client forest companies can also help expand the pool of available, skilled operators by budgeting for competitive wages during pricing negotiations with the contractors.

Being a study based on interviews, some of the time-consumption figures are anecdotal and thus potentially erroneous. However, the figures were provided by the contractors themselves, so any errors are probably small. Moreover, figures for key characteristics like worksites and seedling logistics were double-checked with client company foresters.

We identified key factors for intermittently advancing planting machines, but they are probably relevant as well for continuously advancing planting machines. Because of their higher productivities, continuously advancing planting machines will most likely become dominant in the future in Fennoscandia (despite the prevalence of moraine/glacial till soils in the region) $[2,3,21,41]$.

The cost-competitiveness of mechanized tree planting is certainly reliant on the cost-efficiency of the alternatives, specifically manual tree planting following mechanical site preparation [18]. However, further technical development of Fennoscandian manual tree planting has been judged to be poor [10], and the future supply of labor for manual planting is predicted to shrink [21], so significant cost decrease of the alternatives is unlikely. Additionally, any technical development regarding mechanical site preparation can potentially be transferred to planting machines as well [42].

Our identified key factors pave the way for future studies and development. For example, there is the need in both countries for decision support tools to select sites (based on rockiness/stoniness, etc.) and for tools to simplify quality management (e.g., follow-up planting using systems similar to ASTA). Such tools deserve to be developed and further studied. Considering the amount of time spent by contractors on seedling handling/tending (Table 3) and reloading [35], present-day seedling production and delivery methods are not good enough for today's intermittently advancing machines, and definitely not for future continuously advancing machines [43]. Thus, there is continued need for development of machine-specific seedlings and seedling packaging systems. Likewise is the need for continued technical development to increase the machines' productivity.

\section{Conclusions}

According to our observations, there are several well-known factors like the low Capacity Utilization and productivity of today's planting devices, which prevent the cost-competitiveness and growth of the Fennoscandian mechanized tree planting business. But there are also some factors that cannot be addressed by one counterpart alone. The availability of skilled operators and efficient seedling logistics are two such bottlenecks, which must be solved cooperatively.

In both countries, a large-scale breakthrough of mechanized planting is still waiting to happen. Neither in Finland nor in Sweden was mechanized planting on a routine-level similar to other forestry contracting. Indeed, there are many operational processes, which can be considered to be in their developmental infancy. This includes the process of choosing the work sites for mechanized planting, as well as the process of delivering seedlings throughout the whole planting season. Because mechanized planting in Sweden and Finland share many similar challenges, cooperation between the countries' forest industries and research institutes is both desirable and needed, if tree planting is to become mechanized in Fennoscandia.

Author Contributions: B.T.E. conceived and designed the experiments; B.T.E. and T.S. performed the experiments; B.T.E. and T.S. analyzed the data; B.T.E., T.L., and T.S. wrote the paper.

Acknowledgments: SLU and Luke provided the funding for this study. The travel costs for data gathering was funded by NordGen Forest-SNS scholarships 2017. 
Conflicts of Interest: The authors declare no conflict of interest. The founding sponsors had no role in the design of the study; in the collection, analyses, or interpretation of data; in the writing of the manuscript, and in the decision to publish the results.

\section{References}

1. Bäckström, P.-O.; Jansson, E.; Jeansson, E.; Sirén, G. Comparative Study of Four Tree-Planting Machines; Rapporter och Uppsatser Nr 21; Institutionen för Skogsföryngring; Skogshögskolan: Stockholm, Sweden, 1970.

2. Ersson, B.T. Concepts for Mechanized Tree Planting in Southern Sweden. Ph.D. Thesis, SLU, Umeå, Sweden, 2014.

3. Laine, T. Mechanized Tree Planting in Finland and Improving its Productivity. Ph.D. Thesis, University of Helsinki, Helsinki, Finland, 2017.

4. Hallongren, H.; Laine, T.; Rantala, J.; Saarinen, V.-M.; Strandström, M.; Hämäläinen, J.; Poikela, A. Competitiveness of mechanized tree planting in Finland. Scand. J. For. Res. 2014, 29, 144-151. [CrossRef]

5. Ersson, B.T.; Petersson, M. Återinventering av 2010 års Maskinplanteringar-3-års Uppföljning. [Three-Year Follow-up of 2010's Mechanically Planted Seedlings]; Rapport S048; Skoglig Service; Södra Skog: Växjö, Sweden, 2013.

6. Luoranen, J.; Viiri, H. Deep planting decreases risk of drought damage and increases growth of Norway spruce container seedlings. New For. 2016, 47, 701-714. [CrossRef]

7. Ersson, B.T.; Jundén, L.; Lindh, E.M.; Bergsten, U. Simulated productivity of conceptual, multi-headed tree planting devices. Int. J. For. Eng. 2014, 25, 201-213. [CrossRef]

8. Laine, T.; Kärhä, K.; Hynönen, A. A survey of the Finnish mechanized tree-planting industry in 2013 and its success factors. Silva Fenn. 2016, 50. [CrossRef]

9. Lindholm, E.-L.; Berg, S. Energy Use in Swedish Forestry in 1972 and 1997. Int. J. For. Eng. 2005, 16, $27-37$.

10. Hallonborg, U.; von Hofsten, H.; Mattson, S.; Hagberg, J.; Thorsén, Å.; Nyström, C.; Arvidsson, H. Maskinell mlantering med Silva Nova -nuvarande status samt utvecklingsmöjligheter i jämförelse med manuell plantering [Mechanized Planting with the Silva Nova Tree Planter-Recent State and Feasibility Compared with Manual Planting]; Redogörelse Nr 6; Skogforsk: Uppsala, Sweden, 1995.

11. LUKE. Statistics Database: Forest Statistics: Structure and Production: Silvicultural and Forest Improvement Work; LUKE: Helsinki, Finland, 2018.

12. Rantala, J.; Laine, T. Productivity of the M-Planter Tree-Planting Device in Practice. Silva Fenn. 2010, 44, 859-869. [CrossRef]

13. Ersson, B.T.; Junden, L.; Bergsten, U.; Servin, M. Simulated productivity of one- and two-armed tree planting machines. Silva Fenn. 2013, 47. [CrossRef]

14. Laine, T.; Rantala, J. Mechanized tree planting with an excavator-mounted M-Planter planting device. Int. J. For. Eng. 2013, 24, 183-193. [CrossRef]

15. Rantala, J.; Harstela, P.; Saarinen, V.-M.; Tervo, L. A techno-economic evaluation of Bracke and M-Planter tree planting devices. Silva Fenn. 2009, 43, 659-667. [CrossRef]

16. St-Amour, M. Reforestation Trials with the Bräcke P11.a Planter; Advantage Report Volume 11, No. 19; FPInnovations-FERIC: Pointe-Claire, QC, Canada, 2009.

17. Von Hofsten, H. Hög kvalitet även på högkvaliteten med Öje-Planter [The Öje Planter Machine-Good Performance at a Competitive Cost]; Resultat NR 3; Skogforsk: Uppsala, Sweden, 1993.

18. Ersson, B.T. Possible Concepts for Mechanized Tree Planting in Southern Sweden-An Introductory Essay on Forest Technology; Arbetsrapport 269; Department of Forest Resource Management, SLU: Umeå, Sweden, 2010.

19. Ersson, B.T.; Bergsten, U.; Lindroos, O. The cost-efficiency of seedling packaging specifically designed for tree planting machines. Silva Fenn. 2011, 45, 379-394. [CrossRef]

20. Sønsteby, F.; Kohmann, K. Forsøk med maskinell planting på Østlandet [Mechanized Planting Trials in Østlandet]; Oppdragsrapport 3/03; Norsk Institutt for Skogforskning: Ås, Norway, 2003.

21. Strandström, M.; Hämäläinen, J.; Pajuoja, H. Metsänhoidon koneellistaminen-Visio ja T\&K-ohjelma [The Mechanization of Forestry-Vision and RED Program]; Metsätehon Raportti 206; Metsäteho: Helsinki, Finland, 2009.

22. Luoranen, J.; Rikala, R.; Smolander, H. Machine planting of Norway spruce by Bracke and Ecoplanter: An evaluation of soil preparation, planting method and seedling performance. Silva Fenn. 2011, 45, 341-357. [CrossRef] 
23. Ersson, T. Review of Transplanting and Seedling Packaging Systems in Swedish Tree Nurseries; Technical Report; FPInnovations: Pointe Claire, QC, Canada, 2015; p. 22.

24. Nilsson, U.; Luoranen, J.; Kolström, T.; Örlander, G.; Puttonen, P. Reforestation with planting in northern Europe. Scand. J. For. Res. 2010, 25, 283-294. [CrossRef]

25. Metsäteho. Koneistuttaja palvelu [Machine Service]; Metsäteho: Helsinki, Finland, 2017.

26. Berg, S. Terrängtypschema [Terrain Classification System for Forestry Work]; Forskningsstiftelsen Skogsarbeten: Stockholm, Sweden, 1982.

27. Kärhä, K.; Hynönen, A.; Laine, T.; Strandström, M.; Sipilä, K.; Palander, T.; Rajala, P.T. Koneellinen metsänistutus ja sen tehostaminen Suomessa [Mechanized Planting in Finland and Its Enhancement]; Report NR 233; Metsäteho: Vantaa, Finland, 2014; p. 42.

28. Risutec. ASTA Documentation System. Available online: http://www.risutec.fi/en/products/softwood/asta (accessed on 12 March 2018).

29. Malinen, J.; Laitila, J.; Väätäinen, K.; Viitamäki, K. Variation in age, annual usage and resale price of cut-to-length machinery in different regions of Europe. Int. J. For. Eng. 2016, 27, 95-102. [CrossRef]

30. Spinelli, R.; Magagnotti, N.; Picchi, G. Annual use, economic life and residual value of cut-to-length harvesting machines. J. For. Econ. 2011, 17, 378-387. [CrossRef]

31. Ackerman, P.; Gleasure, E.; Ackerman, S.; Shuttleworth, B. Standards for Time Studies for the South African Forest Industry; ICFR/FESA: Scottsville, South Africa, 2014; p. 49.

32. Erlandsson, E.; Fjeld, D. Impacts of service buyer management on contractor profitability and satisfactionA Swedish case study. Int. J. For. Eng. 2017, 28, 148-156. [CrossRef]

33. Mäkinen, P. Metsä-ja Puualan pk-Yritysten Menestystekijät [Success Factors for SMEs in Forestry and Woodworking]; Finnish Forest Research Institute Research Papers 869; Metla: Vantaa, Finland, 2002; pp. 1-52.

34. Mäkinen, P. Success Factors for Forest Machine Entrepreneurs. J. For. Eng. 1997, 8, 27-35.

35. Ersson, B.T.; Bergsten, U.; Lindroos, O. Reloading mechanized tree planting devices faster using a seedling tray carousel. Silva Fenn. 2014, 48. [CrossRef]

36. Eriksson, M. Developing Client-Supplier Alignment in Swedish Wood Supply. Ph.D. Thesis, SLU, Umeå, Sweden, 2016.

37. Lindroos, O. Scrutinizing the Theory of Comparative Time Studies with Operator as a Block Effect. Int. J. For. Eng. 2010, 21, 20-30.

38. Purfürst, F.T.; Erler, J. The Human Influence on Productivity in Harvester Operations. Int. J. For. Eng. 2011, 22, 15-22.

39. Ovaskainen, H. Timber harvester operators' working technique in first thinning and the importance of cognitive abilities on work productivity. Diss. Fore. 2009. [CrossRef]

40. Valinger, K. Skogsbrukets Framtida arbetskraftsförsörjning-Skogsmaskinföraryrkets attraktionskraft [The Future Labour in Swedish Forestry - The Attraction of Forest Machine Operation as a Profession]; Arbetsrapport 244; Institutionen för Skoglig Resurshushållning, SLU: Umeå, Sweden, 2009.

41. Malmberg, C.-E. Mekanisering av skogsodling [The Mechanization of Forest Cultivation]; STU-info 783-1990; Styrelsen för Teknisk Utveckling: Stockholm, Sweden, 1990; p. 196.

42. Berg, S. Studier av mekaniserade system för markberedning och plantering. [Studies of Mechanized Systems for Scarification and Planting]; Meddelande Nr 19; Forskningsstiftelsen Skogsarbeten: Kista, Sweden, 1991.

43. Saarinen, V.-M.; Hyyti, H.; Laine, T.; Strandström, M. Kohti jatkuvatoimista koneistutusta. [Towards Continuously Operating Planting Machines]; Metsätehon Raportti 227; Metsäteho: Vantaa, Finland, 2013.

(C) 2018 by the authors. Licensee MDPI, Basel, Switzerland. This article is an open access article distributed under the terms and conditions of the Creative Commons Attribution (CC BY) license (http://creativecommons.org/licenses/by/4.0/). 